\title{
Théories et pratiques en développement territorial : des grands projets aux initiatives locales et vice versa
}

\author{
Martin Simard, Thierno Diallo et Suzanne Tremblay ${ }^{1}$
}

\section{INTRODUCTION}

\begin{abstract}
Au Québec, au Canada et ailleurs dans le monde, l'implantation de grands projets représente l'élément moteur de l'économie de marché depuis plusieurs décennies, voire depuis plus d'un siècle.
\end{abstract}

Les divers types de territoires font face aux défis constants du développement social et économique. Tous les milieux géographiques sont interpellés par ces enjeux, des grandes agglomérations urbaines aux régions rurales périphériques, des espaces nordiques à la savane africaine. Les analyses sur les problèmes de développement identifient fréquemment les faiblesses institutionnelles et administratives ou le climat politique sur un territoire donné. Néanmoins, la géographie et les ressources naturelles doivent également être considérées comme étant des facteurs significatifs à considérer ${ }^{2}$. La mise en valeur des ressources naturelles se fait souvent par l'intermédiaire de grands projets ou de mégaprojets. Comprendre le fonctionnement et les effets de ces projets se révèle donc d'une grande importance dans le champ du développement territorial.

Au Québec, au Canada et ailleurs dans le monde, l'implantation de grands projets représente l'élément moteur de l'économie de marché depuis plusieurs décennies, voire depuis plus d'un siècle. Malgré la montée des initiatives de développement endogène ou local dans les années 1980 et 1990, les grands projets ont encore un poids considérable dans l'économie. On observe même un retour de ceux-ci, notamment dans les régions périphériques, avec la multiplication de ce type de projets, en particulier dans le secteur minier. Dans une large mesure, cette situation est associée au phénomène de la mondialisation et à l'expansion des économies émergentes, comme celle de la Chine, lesquelles économies stimulent la demande en ressources naturelles à l'échelle de la planète. Cette forte demande amène à son tour une hausse des prix des matières premières et la mise en exploitation d'un grand nombre de gisements miniers.

Il est difficile de définir exactement ce qu'est un grand projet. De manière générale, il est permis d'affirmer qu'il s'agit d'un projet industriel ou d'infrastructures qui vise à mettre en place des équipements de production de grande taille. Ces équipements se démarquent par l'ampleur des ressources mobilisées et des investissements, tout en comportant des défis de coordination d'une série d'acteurs publics ou privés sur plusieurs mois ou années. De par son envergure, ce genre de projets est susceptible d'avoir des retombées majeures sur les communautés ou collectivités d'accueil, retombées qui peuvent être positives ou négatives (emplois, aménités, impacts sociaux ou environnementaux, etc.). Si la définition des grands projets est plutôt fluide, notamment en ce qui concerne le seuil à partir duquel l'on peut parler de ce type de projets, la terminologie pose également problème. En effet, doit-on parler de grands projets ou de mégaprojets? Les deux mots peuvent être utilisés comme synonymes, mais nous avons une préférence pour l'expression «grand projet ».

Par ailleurs, la question de la pertinence ou de l'efficacité des grands projets mérite notre attention. Les théories économiques classiques font la promotion des grands projets et soulignent leurs influences sur la croissance et le développement, dans une optique territoriale ou plus générale ${ }^{3}$. Néanmoins, dans plusieurs cas, la rentabilité économique n'est pas au rendez-vous, ou du moins, celle-ci émerge sur la longue durée, alors que l'accent est fréquemment placé sur la 
« rentabilité politique », en particulier dans le cas des projets publics. Certains auteurs affirment même que les petits projets initiés par des petites et moyennes entreprises (PME) participent davantage au développement, voire que les grands projets sont carrément nuisibles pour les collectivités locales et régionales ${ }^{4}$.

Ce texte vise à traiter des changements de paradigmes en développement régional. Plus précisément, il s'agit d'illustrer le retour des grands projets à l'aide de deux exemples affichant des similarités, soit le Nord québécois et l'Afrique subsaharienne. Nous aimerions d'abord discuter de ce qui semble constituer un virage significatif dans les théories et les stratégies dans le champ du développement territorial, soit le retour des grands projets de développement depuis le début de ce millénaire. Dans un deuxième temps, nous traiterons des grands projets dans deux milieux spécifiques, soit le Nord du Québec et le continent africain.

\section{LE DÉVELOPPEMENT LOCAL : UN PARADIGME EN VOIE DE MARGINALISATION?}

\section{L'origine et les formes du développement local}

Le développement endogène met de l'avant

la prise en compte des besoins fondamentaux des populations en lien avec les spécificités des territoires et de leurs différentes composantes, plus précisément les composantes naturelle, économique, culturelle, et sociale.

Le virage actuel semble suivre un premier tournant opéré il y a une trentaine d'années dans le champ du développement territorial, c'est-à-dire la montée du paradigme du développement local. L'approche du développement local est apparue au cours des années 1980, après plusieurs décennies de développement économique impulsé par le haut, soit par l'État ou la grande industrie. Cette vague initiale de développement, qui fut à son sommet dans les «Trente Glorieuses », focalisait sur les grands projets, qu'il s'agisse de mines, de barrages hydroélectriques, de centrales nucléaires ou de complexes industriels dans divers secteurs d'activités (sidérurgie, pétrochimie, pâtes et papiers, aluminium, automobile, aéronautique, etc.). À la suite de cette période faste, le développement local s'est progressivement imposé sur plusieurs plans dont le plan théorique, le plan politique de même qu'au sein des communautés locales et régionales elles-mêmes.

Sur le plan théorique, le développement local est apparu dans le sillage du développement endogène. Le développement endogène se veut une approche territoriale du développement et celle-ci relève du paradigme du développement par le bas. Le développement endogène met de l'avant la prise en compte des besoins fondamentaux des populations en lien avec les spécificités des territoires et de leurs différentes composantes, plus précisément les composantes naturelle, économique, culturelle, et sociale. Le développement endogène a fait l'objet de nombreuses publications savantes de la part de plusieurs auteurs, tant des économistes, que des géographes ou des sociologues ${ }^{5}$. Plus profondément, ce mouvement est issu de multiples courants théoriques qui se sont mis en place à la fois dans les pays du Sud et dans les pays du Nord. Pour certains auteurs, le développement local est synonyme de développement endogène alors que d'autres $\mathrm{y}$ voient des distinctions subtiles sur lesquelles nous ne nous attarderons pas.

De manière synthétique, disons que deux grandes approches de développement local se dégagent et que celles-ci englobent les différents courants: l'approche du développement économique local et celle du développement économique communautaire. Selon les différentes versions du développement local et selon les auteurs qui le décrivent ${ }^{6}$, l'accent sera mis sur le développement économique ou le développement social. Cependant, le territoire local et sa population sont toujours au centre de l'équation. Ainsi, le développement local mobilise inévitablement les institutions locales et implique des formes de décentralisation des pouvoirs publics. Des groupes de citoyens plus informels peuvent également contribuer au processus. D'une certaine façon, il s'agissait de la rencontre historique d'une demande venant de la base (populations, groupes, municipalités locales ou MRC, etc.) et d'une offre émanant du haut (gouvernements supérieurs). 
L'approche du développement économique local (DEL) met l'accent sur les indicateurs traditionnels du développement, soit la création d'emplois, la croissance des revenus et du PIB à partir d'initiatives mises en œuvre notamment par les acteurs du secteur privé, en particulier des PME. Certains auteurs parlent de modèle fonctionnel qui se traduit par une tentative d'ajustement aux marchés mondialisés. Dans cette perspective, selon Bernard Vachon, le développement économique local se veut une stratégie basée sur la synergie des partenaires afin de mettre en œuvre des initiatives locales et «d'augmenter la capacité des collectivités en difficulté à s'adapter aux nouvelles règles du jeu de la croissance macroéconomique; ou à trouver d'autres formes de développement » $[. .]$.$» intégrant à la fois «des$ préoccupations d'ordre social, culturel et environnemental parmi des considérations purement économiques $\gg^{7}$.

Lorsqu'il est question de développement économique local, l'accent est généralement placé sur la collaboration des acteurs dans une optique de développement à caractère économique, mais les aspects social, culturel et environnemental peuvent être présents à différents degrés. Cette forme de développement local se décline en plusieurs sous-courants plus ou moins formalisés, par exemple l'approche des districts industriels, celle des milieux innovateurs, la critique postfordiste ou la théorie de la spécialisation flexible des systèmes productifs locaux $^{8}$.

Pour ce qui est du développement économique communautaire (DEC), il est, selon Martine D'Amours, une composante du développement local, mais, à la différence de celui-ci, il intègre «des objectifs économiques (création d'entreprises et d'emplois) et des objectifs sociaux (lutte contre la marginalisation sociale, qualité de vie, services collectifs, empowerment de la communauté) dans une approche globale » et avec une stratégie territoriale ${ }^{9}$. Le DEC tend à prendre en compte quatre dimensions, soit la dimension économique par la production et la vente de biens et services, la dimension territoriale par la mise en valeur des ressources locales, la dimension sociale par un travail portant sur la recomposition sociale de la communauté et la dimension communautaire « en tant qu'espace du vivre ensemble et en tant que dispositif premier de revitalisation ${ }^{10}$.

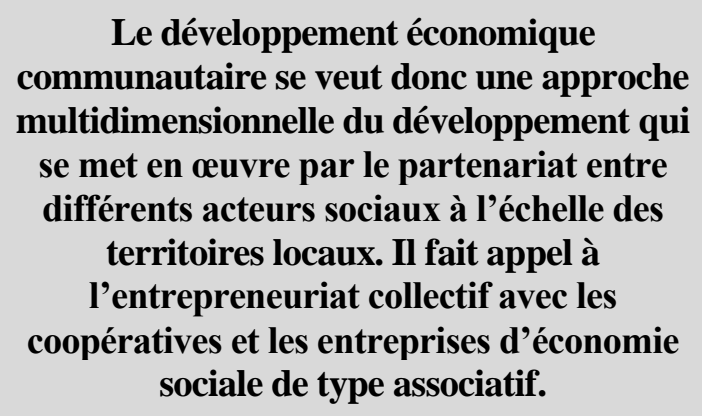

Le développement économique communautaire se veut donc une approche multidimensionnelle du développement qui se met en œuvre par le partenariat entre différents acteurs sociaux à l'échelle des territoires locaux. Il fait appel à l'entrepreneuriat collectif avec les coopératives et les entreprises d'économie sociale de type associatif.

Le développement économique communautaire se veut donc une approche multidimensionnelle du développement qui se met en œuvre par le partenariat entre différents acteurs sociaux à l'échelle des territoires locaux. Il fait appel à l'entrepreneuriat collectif avec les coopératives et les entreprises d'économie sociale de type associatif. Le DEC prend racine à partir des besoins de la communauté ainsi que de la volonté des acteurs sociaux à s'engager dans une dynamique de développement territorial, social, communautaire et économique. Dans cette perspective, l'action des réseaux d'acteurs apparaît au cœur de tout processus de développement local. Cette perspective du développement local correspond à une vision sociale des interventions à l'échelle des collectivités territoriales.

\section{Politiques publiques, développement local et dynamiques d'acteurs}

Le développement local a été décrit par plusieurs chercheurs, mais l'engouement pour cette approche s'est également concrétisé sur le plan politique au Québec notamment au début des années 1980. En effet, avec la crise économique du début des années 1980 et plus largement avec la crise du fordisme, les États des pays occidentaux ont dû procéder à un délestage de leurs responsabilités. De ce fait, les États ont connu une perte de légitimité comme acteur central au profit d'une redistribution des rôles de régulation entre les différentes composantes de la société. Dans ce contexte, l'État québécois est devenu accompagnateur et le développement local s'est avéré être 
une prescription pour pallier les problèmes du désengagement étatique. L'État a donc transféré aux administrations locales et supralocales la tâche de gérer et d'absorber les effets de son déclin relatif.

Pendant la période allant du début des années 1980 jusqu'aux années 2000, plusieurs politiques publiques reliées de près ou de loin au développement local et régional ont été adoptées par les gouvernements successifs au Québec. À cet égard, on peut premièrement nommer la loi sur l'aménagement et l'urbanisme adoptée en 1979, laquelle a créé un palier administratif à l'échelle microrégionale ou supralocale, soit les municipalités régionales de comté (MRC). Deuxièmement, nous citerons l'énoncé de politique intitulé «Le choix des Régions » publié en 1983. Troisièmement, il importe de mentionner le lancement de «La politique de soutien au développement local et régional » en 1997. Finalement, il est permis de souligner diverses politiques plus récentes qui s'inspirent du développement local : la Loi sur les regroupements municipaux (2000), la Politique nationale de l'eau (2001), la Politique nationale de la ruralité (2002 et 2008), la Stratégie pour assurer l'occupation et la vitalité des territoires (2011), etc.

Avec toutes ces politiques, stratégies ou programmes, l'État québécois a fait des acteurs locaux les pivots de la mise en œuvre du développement local. Cependant, l'État central est demeuré omniprésent dans ces politiques puisque c'est lui qui définit et oriente les politiques de développement local. Autrement dit, la mise en œuvre du développement local et des politiques qui y sont associées sont demeurés sous la coordination du gouvernement provincial à l'intérieur d'un mode de gouvernance souple et flexible.

Malgré l'omniprésence de l'État, la mise en œuvre du développement local a été reprise par les acteurs locaux, notamment par le milieu de l'entrepreneuriat avec la participation à la mise en place de territoires spécialisés selon des créneaux d'excellence. De plus, les acteurs de la société civile se sont révélés comme étant des coproducteurs du développement économique communautaire et de l'économie sociale. Ainsi, le discours sur l'importance du local n'est pas venu exclusivement des réformes étatiques, mais il a pris forme au niveau même des collectivités locales et des acteurs locaux. En effet, la dévitalisation de plusieurs communautés rurales, et aussi de certains quartiers urbains, a incité les acteurs des communautés locales à œuvrer pour la prise en main de leur milieu. Dans cette perspective, les initiatives de développement local se sont multipliées tant en milieu rural qu'en milieu urbain et c'est ainsi que le développement économique local et le développement économique communautaire sont devenus des outils largement répandus pour favoriser l'autonomisation des collectivités locales.

Le développement local prescrit par l'État a donc été repris par les acteurs sociaux à la base qui cherchent à pallier les effets des crises économiques, de la réduction de la taille de l'État et de la dévitalisation des milieux tant urbains que ruraux. Pour certains, le développement local ne représente qu'une collection d'initiatives locales éparses qui s'apparentent davantage à une tradition de pratiques qu'à un véritable modèle de développement ${ }^{11}$. Pour d'autres cependant, le développement local représente la base d'un véritable modèle de développement centré sur l'initiative locale et sur la coopération des acteurs territoriaux. Dans cette perspective, le développement local, selon Juan-Luis Klein, «se présente comme le début d'un processus cyclique qui instaure une dynamique de développement basée sur l'initiative locale et la mobilisation d'actifs endogènes et exogènes $»^{12}$. De plus, à compter des années 1990 , certains auteurs font la promotion du développement territorial qui se veut «un paradigme scientifique en émergence » qui englobe le développement local, puisque celui-ci s'inscrit dorénavant sur «une scène multiscalaire et multisectorielle $»^{13}$.

Dans le contexte des années 2000, cependant, le développement local apparaît de moins en moins suffisant pour contrer la dévitalisation des milieux ruraux et urbains de même que la décroissance économique qui touche les pays industrialisés depuis la crise économique de 2008. L'attrait pour les grands projets semble revenir en force dans les officines gouvernementales. L'exploitation à grande échelle des ressources naturelles, notamment des ressources minières et pétrolières semblent être les nouvelles avenues de développement qui sont préconisées par l'État. Le développement 
par le haut initié par l'État et par les grandes multinationales, tant au Québec qu'ailleurs dans le monde, apparait comme le modèle de développement classique, modèle le plus à même de créer de la richesse, de la croissance et du développement.

$\mathrm{Au}$ surplus, le contexte d'hypermobilité que l'on observe présentement amène une partie de la main- d'œuvre à suivre le travail à travers le navettage de courte ou de longue durée (fly in/fly out) plutôt qu'à s'investir dans le développement de leur milieu. À de multiples égards, le retour aux grands projets ressemble à une fuite en avant à l'époque où plusieurs analystes proposent avec conviction un développement plus durable et annoncent la fin du mythe de la croissance illimité $e^{14}$.

\section{LE NORD QUÉBÉCOIS ET LES ALÉAS DU « PLAN NORD »}

Le Nord québécois est l'un de ces milieux géographiques où l'on observe nettement le retour des grands projets. Il ne s'agit pas cette fois de projets hydroélectriques comme ceux de la Manicouagan ou de la Baie-James, mais d'investissements imposants dans le secteur de l'exploitation minière.

Le Nord québécois est l'un de ces milieux géographiques où l'on observe nettement le retour des grands projets. Il ne s'agit pas cette fois de projets hydroélectriques comme ceux de la Manicouagan ou de la Baie-James, mais d'investissements imposants dans le secteur de l'exploitation minière. En mai 2011, le gouvernement du Québec a annoncé la mise en œuvre d'une stratégie de développement sous le vocable du «Plan Nord». Le Plan Nord est un plan d'action économique qui vise à exploiter davantage les ressources des régions nordiques de la province, en particulier les ressources minières. Le plan proposait quatre objectifs stratégiques pour favoriser le développement des territoires québécois situés au nord du $49^{\mathrm{e}}$ parallèle : assurer le mieux-être et le développement des communautés, mettre en valeur l'immense potentiel économique du Nord québécois, rendre le nord accessible et veiller à la protection de l'environnement. Après une courte période d'enthousiasme marquée par l'annonce de divers investissements privés, le plan a perdu de son élan avec la baisse significative du prix du minerai de fer, entre 2012 et 2014, et le changement de régime politique à Québec, en 2013.

Le gouvernement du Parti québécois a tenté de relancer ce programme de développement sous l'expression «le Nord pour tous», mais les milieux d'affaires ont semblé bouder ce programme modifié. Le retour au pouvoir des libéraux en avril 2014 pourrait signifier une relance du Plan Nord en dépit du contexte d'austérité budgétaire. Néanmoins, le succès de cette politique de développement régional dépend largement des actions du secteur privé et du contexte économique international. De plus, il importe de rappeler que le nord du Québec est immense et que les problèmes de transports et de communications y sont énormes. La trentaine de villes et villages qu'on y retrouve constituent de petits isolats situés en dehors de l'écoumène québécois. En outre, ces établissements éloignés sont également distants les uns des autres, établissements que l'on ne peut souvent atteindre que par avion ou par transport maritime, lors de la saison estivale. Le froid, les eaux englacées et le pergélisol limitent passablement la faisabilité et la pérennité des travaux d'aménagement et laissent l'environnement très vulnérable face aux éventuelles catastrophes.

La question des droits et revendications autochtones est aussi à considérer. Les territoires nordiques du Québec sont pour la plupart gérés par le biais de la convention de la Baie-James et du nord-est québécois. Celle-ci stipule notamment que les droits ancestraux des signataires sont éteints et elle offre beaucoup de marge de manœuvre au gouvernement du Québec en dehors des terres de catégories I et II. Toutefois, la question est davantage politique que juridique. Jusqu'à maintenant, les Cris de la Jamésie (région aussi appelée Eeyou Istchee) ont réagi favorablement au projet, mais qu'en sera-t-il des autres nations? Certains groupes d'Innus ont d'ailleurs exprimé leur mécontentement face au Plan Nord. Rappelons que ceux-ci vivent principalement dans la région administrative de la Côte-Nord, territoire qui n'est pas conven- 
tionné, c'est-à-dire que les droits territoriaux des premières nations ne sont pas précisément balisés sur le plan légal. De plus, les négociations sur «l'approche commune» piétinent depuis plus d'une décennie.

Par ailleurs, la plupart des mines de fer, de zinc ou de nickel qui seront exploitées ne donneront pas lieu à de nouvelles villes ou de nouveaux villages. Les dirigeants favoriseront certainement les déplacements temporaires des travailleurs, à partir des villes du sud du Québec. C'est ce qui est fait à la mine de nickel Raglan, implantée en 1997 dans la péninsule de l'Ungava. Du côté des établissements «blancs », Fermont pourrait voir sa population augmenter avec la mise en œuvre des projets miniers actuellement sur la planche à dessin dans le secteur de la fosse du Labrador. Schefferville devrait également redevenir un pôle de développement considérant sa localisation stratégique au cœur du Québec nordique, la présence d'infrastructures ainsi que ses populations autochtones. D'ailleurs, les projets miniers de la compagnie New Millenium situés dans ce secteur se développent en bonne partie grâce à l'apport de travailleurs innus et naskapis recrutés sur les réserves de Matimekosh-Lac-John et de Kawawa- chikamach, qui sont à proximité de Schefferville. Néanmoins, le phénomène du fly in/fly out devrait réduire énormément les possibilités d'expansions de ces petites villes isolées. Une grande partie des retombées risque aussi de se réaliser du côté terreneuvien du Labrador.

Sur le plan législatif, l'adoption d'une nouvelle loi sur les mines en décembre 2013 précise le contexte dans lequel les minières doivent opérer, après quelques années d'incertitudes. Toutefois, des projets publics contenus dans le Plan Nord, soit un lien routier ou ferroviaire à partir du sud pour rejoindre Kuujjuaq, sur la Baie d'Ungava; un port en eau profonde à Kuujjuarapik, sur la Baie D'Hudson, se font toujours attendre. Des investissements publics de près de 400 millions avaient aussi été prévus dans les sphères du logement, de la santé et de l'éducation dans le Plan Nord de 2011. Est-ce qu'ils seront relancés par le nouveau gouvernement à Québec? Finalement, les ententes secrètes entre les communautés autochtones et les compagnies minières ou Hydro-Québec sont dénoncées par de nombreux groupes, incluant des Autochtones, groupes qui réclament plus de transparence à ce niveau.

\section{LES GRANDS PROJETS MINIERS EN CONTEXTE AFRICAIN}

\section{Les exigences des États et les retombées locales}

\section{Le continent africain est un autre territoire}

riche en minerais qui est touché par la vague de grands projets, que ce soit en milieu désertique, sahélien ou dans les zones de forêt tropicale.

Le continent africain est un autre territoire riche en minerais qui est touché par la vague de grands projets, que ce soit en milieu désertique, sahélien ou dans les zones de forêt tropicale. Selon diverses estimations, l'Afrique détiendrait un tiers des réserves mondiales, tous minerais confondus. Forts de ces richesses et pour attirer les investisseurs étrangers, les pays africains ont adopté des codes d'investissements extrêmement généreux (exemptions fiscales, avantages douaniers, etc.). Ainsi, avec ces politiques attractives et l'accroissement de la demande mondiale de certains minerais depuis la fin du $X X X^{\mathrm{e}}$ siècle, de nombreux investisseurs étrangers ont ciblé le continent africain et depuis les grands projets miniers se sont multipliés. Les entreprises minières canadiennes ont particulièrement profité de l'ouverture de ces nouveaux marchés et de nombreux contrats miniers furent signés dans divers pays d'Afrique.

De nos jours, sous diverses pressions, les compagnies minières en Afrique initient de plus en plus des actions en faveur du développement local des zones qui accueillent les différents projets. Ainsi, les grands projets et le développement local ne sont pas incompatibles même s'il ne s'agit pas purement d'initiatives issues du milieu. Afin de promouvoir le développement local des zones minières, les sociétés minières versent chaque année un montant déterminé aux collectivités territoriales sur une base contractuelle ou sur la base d'une entente entre la société minière et l'État. Par ailleurs, ces sociétés minières, à travers des fondations qu'elles ont mises en place, investissent des fonds assez importants pour 
appuyer le développement communautaire dans les zones de production en finançant des projets communautaires dans le but de maintenir un climat favorable et de sécuriser les importants investissements réalisés et l'exploitation subséquente.

Par exemple au Mali, conformément à l'article 125 du Code minier de 1999 qui prévoit plusieurs obligations pour les entreprises exploitant une mine en matière de développement local. Par exemple, la mine d'or de Morila S.A. a initié en 2000 un fonds de développement communautaire dans le cadre de son programme de développement local ${ }^{15}$. Dès lors, ce fonds sert à la construction de plusieurs édifices dans les quatre communes à proximité de la mine: des salles de classe, des écoles, des latrines, des puits, des dispensaires. Par ailleurs, une somme de 30000 \$ US est dépensée chaque année pour apporter un soutien à la gendarmerie (en salaires ou en infrastructure) pour la protection des investissements. Le fonds de développement local a également permis l'électrification de la mairie, de la place publique, de la maternité et du centre de formation des adultes de certains villages proches de la zone de production.

$\mathrm{Au}$ Burkina Faso et au Niger, la compagnie canadienne SEMAFO participe activement au processus de développement local avec plusieurs projets locaux en cours de réalisation. Il s'agit des projets suivants: mise en place de cantines scolaires, mise en place de plateformes multifonctionnelles (PTFM), production et transformation de beurre de karité, production de sésame, prévention du cancer du col de l'utérus, soutien aux femmes atteintes de fistules (Burkina Faso) et du projet de production de paprika (Niger). Au Liberia, après l'élimination des clauses qui affranchissaient le groupe Arcelor Mittal, le gouvernement a obtenu le respect des règles de protection de l'environnement et a imposé l'indemnisation des populations déplacées. Il en va de même pour la Tanzanie qui, grâce à une bonne négociation des contrats miniers, a pu obtenir le paiement de redevances annuelles substantielles par les compagnies minières telles que la Sud-Africaine Anglogold, la Canadienne Barrick ou l'Australienne Resolute Mining Limited. Cette entente a aussi prévu la mise en place d'un fonds spécial destiné à financer des projets au profit des populations vivant dans les zones d'exploitation ${ }^{16}$.

\section{Les problèmes locaux liés à la gestion des grands projets miniers en Afrique}

De nos jours, malgré les efforts consentis par les sociétés minières en faveur du développement local, leurs interventions sont sous-estimées par les acteurs locaux, vu les nombreux impacts que les projets miniers génèrent. À cela s'ajoutent les faiblesses notoires constatées dans les différentes interventions, lesquelles ont conduits à l'élaboration d'objectifs en vue d'améliorer la situation. Ces objectifs sont les suivants: une meilleure gestion des fonds alloués aux collectivités territoriales, la protection de l'environnement, le développement social et sociétal de même que le processus de révision et de renégociation des contrats miniers enclenchés. Regardons brièvement ces quatre grands objectifs de développement.

\section{En Guinée, les organisations de la société civile et les collectivités locales des zones d'intervention se plaignent de n'avoir aucune information sur les critères qui permettent de fixer les montants des fonds alloués par les sociétés minières aux collectivités locales qui accueillent les projets miniers.}

Premièrement, on cherche à obtenir une meilleure gestion des fonds attribués aux acteurs locaux. Selon divers faits ou témoignages, les fonds alloués par les sociétés minières aux collectivités territoriales pour le développement sont parfois mal utilisés. Ainsi, au Mali, il a été dévoilé que les populations locales ignoraient l'existence du fonds de développement local mis en place par la mine d'or de Morila $\mathrm{SA}^{17}$. Toujours selon la même source, pour la mine d'or de Sadiola, les autorités locales dénoncent la lenteur dans la mise en place des programmes sociaux qui sont loin d'ailleurs de répondre actuellement aux besoins de la population. En Guinée, les organisations de la société civile et les collectivités locales des zones d'intervention se plaignent de n'avoir aucune information sur les critères qui permettent de fixer les montants des fonds alloués par les sociétés minières aux collectivités locales qui accueillent les projets miniers. Ainsi, les collectivités de grande taille s'attribuent la part la plus importante (entre $40 \%$ et $47 \%$ en moyenne des fonds versés), alors qu'elles ne sont pas directement concernées par les effets nocifs de l'activité extractive. 
En deuxième lieu, l'enjeu de l'environnement devient de plus en plus important. Au Mali, le Code minier de 1999 contient des obligations spécifiques en matière environnementale obligeant notamment les entreprises à fournir une étude d'évaluation environnementale comme condition à l'octroi du permis d'exploitation. Or, dans la pratique, des faiblesses sont constatées et au Mali selon la FIDH (2007), quand la mine d'or de Syama a fermé en 2001, la surprise fut grande en découvrant que le plan de fermeture n'avait pas été intégré au plan de développement général de la mine. Ainsi, les installations ont été laissées à l'abandon, la mine et le lac de boue à l'air libre. Autrement dit, les installations étaient visibles depuis la route qui longe l'ancienne mine et dont l'accès est encore contrôlé par un poste de gendarmerie selon Oxfam.

Troisièmement, le développement social s'impose comme préoccupation. Certes, l'activité minière crée des richesses, comme c'est le cas au Mali, où les sociétés minières et leurs sociétés de soustraitance ont créé à peu près 11958 emplois. De plus, les salaires versés par les sociétés minières sont passés de 30 millions de dollars US en 2008 à 61 millions de dollars US en 2012, soit une augmentation de $100 \%$ en seulement cinq ans ${ }^{18}$. Néanmoins, les impacts sociaux de l'activité extractive sont complexes. On assiste à l'appropriation des terres des communautés locales et à des déplacements forcés, à des impacts sur la santé, à la modification des rapports sociaux, à la destruction des moyens de subsistance et des modes de vie des populations, à la désagrégation sociale ainsi qu'à des tensions et conflits parfois violents liés à l'activité minière.

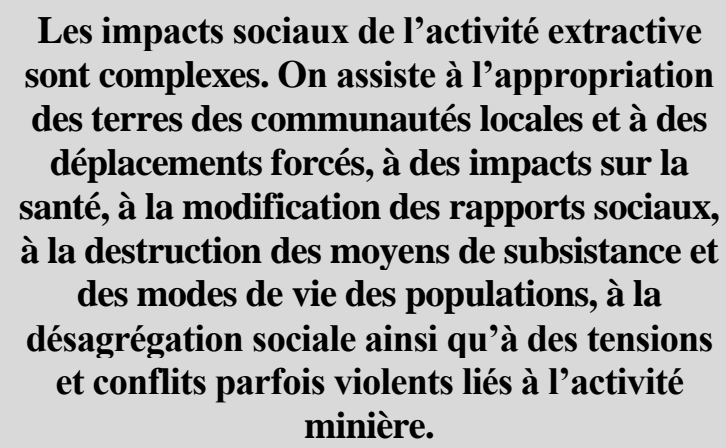

Dans un quatrième temps, la révision des contrats miniers soulève de nombreux défis. Actuellement, face aux besoins subséquents d'améliorer les recettes budgétaires, l'émergence d'une société civile soucieuse de faire participer les populations à la gestion des ressources minières et pétrolières et de leurs revenus et les nouvelles exigences de transparence, les différentes autorités politiques africaines estiment que les bénéfices tirés de l'exploitation des ressources minières ne doivent plus profiter exclusivement aux investisseurs étrangers et aux États. Si la majorité des pays africains miniers ont enclenché des dynamiques de révision et de négociation des contrats miniers, il est clair que ces nouveaux contrats doivent prendre en considération les besoins (environnementaux et sociaux) des populations locales qui sont beaucoup plus affectées par les impacts négatifs de l'activité minière.

\section{Les grands projets miniers et le rôle des acteurs locaux}

Dans l'accompagnement des projets miniers, l'ensemble des acteurs locaux, nationaux et internationaux ont un rôle important à jouer. Ainsi, les collectivités territoriales en plus d'une utilisation judicieuse des fonds qui leur sont versés doivent proposer d'autres alternatives d'emplois aux porteurs de projets locaux. Par ailleurs, elles doivent participer à l'animation des cadres de concertation multiacteurs au niveau local pour échanger sur des enjeux alarmants qui accompagnent la mise en œuvre d'un projet minier (RSE, protection de l'environnement, gestion des conflits, etc.).

Quant à l'État, assurer son rôle de suivi et de contrôle des projets devient capital afin d'harmoniser les différentes interventions sur le terrain. Par ailleurs, il doit faire une meilleure utilisation des retombées et autres aides des sociétés minières pour renforcer les capacités de ces agents d'une part et permettre une redistribution des richesses à travers un investissement sans faille dans les services sociaux de base (éducation, santé, hydraulique).

Enfin, la communauté internationale peut aider les grands projets miniers à travers un appui aux pays 
miniers africains. Il s'agira de renforcer leur capacité à mieux contrôler les activités minières, à fournir une expertise locale appropriée aux mines afin de diminuer les coûts d'expatriation des cadres. Par ailleurs, elle appuiera les États à former des ressources humaines qualifiées afin de négocier des contrats gagnants-gagnants pour les projets futurs. C'est le cas de la Banque africaine de Développement qui a aidé à la création de la

\section{CONCLUSION}

Dans les années à venir, les défis seront nombreux pour la mise en place des grands projets et leur acceptabilité sociale. Dans plusieurs cas, les collectivités locales qui accueillent ces projets ont le sentiment que les entreprises maximisent leur profit au détriment des populations locales et de l'environnement. Autant dans le Nord québécois qu'en milieu africain, l'implantation des projets et l'exploitation subséquente des infrastructures amènent des rencontres entre les communautés qui peuvent être enrichissantes, mais cela augmente également les risques de tensions ou de conflits. Les rétributions ou équipements cédés aux communautés ou acteurs locaux à titre de dédommagement ne constituent pas véritablement des facteurs de développement local et leur gestion soulève des enjeux éthiques et politiques. Ainsi, l'apport de l'extra-local peut faire partie d'une dynamique de développement local ${ }^{19}$, mais, dans le cas des grands projets, le déséquilibre entre les sommes investis et le poids économique et politique des acteurs en présence ne permet pas vraiment à la dynamique de développement d'être maîtrisée à l'échelle locale. Grisant pour les acteurs économiques et politiques, les grands projets n'en constituent pas moins des risques financiers et environnementaux importants. Ces risques sont fréquemment sous-estimés. Une visite à Schefferville permet à quiconque de vérifier les coûts humains et environnementaux des rêves brisés du passé.

\section{BIBLIOGRAPHIE ET NOTES}

${ }^{1}$ Les trois auteurs sont professeurs à l'Université du Québec à Chicoutimi et membres du Centre de recherche sur le développement territorial (CRDT). Ils remercient d'ailleurs cet organisme pour le financement du projet de recherche sur les mégaprojets et le développement territorial.
Facilité africaine de soutien juridique. La Facilité africaine de soutien juridique accompagne depuis 2010 les gouvernements africains dans le cadre de la négociation de transactions commerciales complexes. Elle se propose de remédier à l'asymétrie entre les capacités de négociation respectives des gouvernements africains et des investisseurs internationaux puissants et fortunés.

${ }^{2}$ Sachs, J.D. (2003). «Les institutions n'expliquent pas tout », Finances et développement, $\mathrm{n}^{\circ}$ juin 2003, p. 38-43. ${ }^{3}$ Solow, R.M. (1956). «A Contribution to the Theory of Economic Growth», The Quarterly Journal of Economics, Vol. 70, $\mathrm{n}^{\mathrm{o}}$ 1, p. 65-94 et Rostow, W.W. (1963). «Les étapes de la croissance économique», Paris, Éditions du Seuil, 252 pages.

${ }^{4}$ Julien, P.-A. (2005). Entrepreneuriat régional et économie de la connaissance - une métaphore des romans policiers, Québec, PUQ, 395 pages.

${ }^{5}$ Les nombreux écrits des auteurs suivants: Philippe Aydalot, Michel Bassand, John Friedman, Jean-Louis Guigou, Bernard Planque, Ignacy Sachs, Walter Stöhr, Clyde Weaver, etc.

${ }^{6} \mathrm{D}$ 'Amours, M. (2006). L'économie sociale au Québec, Cadre théorique, histoire réalités et défis, Montréal, Éditions St-Martin, ARUC-ÉS, RQRP-ÉS, 150 pages; Klein, J.L. (2008). Territoire et développement, du local à la solidarité interterritoriale, dans G. Massicotte (dir.), Sciences du territoire, Perspectives québécoises, Québec, PUQ, p. 315-333; Pecqueur, B., (1989). Le développement local: mode ou modèle, Paris, Syros/Alternatives, 149 pages; Vachon, B. (1993). Le développement local. Théorie et pratique, Boucherville, Gaëtan Morin Éditeur, 320 pages.

${ }^{7}$ Voir VACHON, B. (1993) Le développement local. Théorie et pratique, Boucherville, Gaëtan Morin Éditeur, page 104.

${ }^{8}$ Proulx, M.U. (1995). Réseaux d'information et dynamique locale, Chicoutimi, GRIR/UQAC, 334 p.

${ }^{9} \mathrm{D}$ 'Amours, M. (2006). L'économie sociale au Québec, Cadre théorique, histoire réalités et défis, Montréal, Éditions St-Martin, ARUC-ÉS, RQRP-ÉS, 150 pages.

${ }^{10}$ Voir Favreau, L. et Lévesque, B. (1996). Développement économique communautaire. Économie sociale et intervention, Québec, PUQ, page XIX.

${ }^{11}$ Joyal, A. (2012). "Le développement local au Québec: trois décennies de contributions », Revue d'économie régionale et urbaine, $\mathrm{n}^{\mathrm{o}} 4$, p. 673-690.

${ }^{12}$ Klein, J.L. (2008). «Territoire et développement, du local à la solidarité interterritoriale ». Dans G. Massicotte (dir.), Sciences du territoire, Perspectives québécoises, Québec, PUQ, p. 324 
${ }^{13}$ Jean, B. (2008). «Le développement territorial une discipline scientifique émergente », dans Massicotte, G. (dir.), Sciences du territoire, Perspectives québécoises, Québec, PUQ, p. 283-313 et Klein, J.L., 2008, p. 318.

${ }^{14}$ Rubin, J. (2012). The End of Growth, Toronto, Random House, 304 pages.

${ }^{15}$ Fidh (2007). "Mali, l'exploitation minière et les droits humains », mission internationale d'enquête, Fédération internationale des ligues des droits de l'homme, document $\mathrm{n}^{\circ}$ 477, 52 pages.

${ }^{16}$ Fall, A. (2012). «Contrats d'investissement miniers : exorciser la malédiction de l'abondance », Le Quorum, $\mathrm{n}^{\mathrm{o}} 1, \mathrm{p} .7-11$.

${ }^{17}$ Fidh (2007). «Mali, l'exploitation minière et les droits humains ", mission internationale d'enquête, Fédération internationale des ligues des droits de l'homme, document $\mathrm{n}^{\circ} 477,52$ pages.

${ }^{18}$ Source : www.chambredesminesdumali.org

${ }^{19}$ Voir Cote, S. (2003). «Développement local : sur fond de proximité relationnelle, l'apport indispensable de l'extra-local ", Revue Interventions économiques, $\mathrm{n}^{\mathrm{o}} 30$ [En ligne], URL: http://interventions économiques. revues.org/1002, consulté le 21 avril 2014. 